Chapter 11

Adaptive IEEE 802.15.4 Medium Access Control Protocol for Control and Monitoring Applications

\author{
Pangun Park, Carlo Fischione, and Karl Henrik Johansson
}

\begin{abstract}
The IEEE 802.15.4 standard for wireless sensor networks (WSNs) can support energy efficient, reliable, and timely packet transmission by tuning the medium access control (MAC) parameters macMinBE, macMaxCSMABackoffs, and macMaxFrameRetries. Such a tuning is difficult, because simple and accurate models of the influence of these parameters on the probability of successful packet transmission, packet delay, and energy consumption are not available. Moreover, it is not clear how to adapt the parameters to the changes of the network and traffic regimes by algorithms that can run on resource-constrained nodes. In this chapter, a generalized Markov chain is proposed to model these relations by simple expressions without giving up the accuracy. In contrast to previous work, the presence of limited number of retransmissions, acknowledgments, unsaturated traffic, and packet size is accounted for. The model is then used to derive an adaptive algorithm for minimizing the power consumption while guaranteeing reliability and delay constraints in the packet transmission. The algorithm does not require any modification of the IEEE 802.15.4 standard and can be easily implemented on network nodes. Numerical results show that the analysis is accurate and that the proposed algorithm satisfies reliability and delay constraints, and ensures a longer lifetime of the network under both stationary and transient network conditions.
\end{abstract}

Keywords IEEE 802.15.4 • Wireless sensor network - Markov chain model • Optimization

\title{
11.1 Introduction
}

The IEEE 802.15.4 standard has received considerable attention as a low data rate and low power protocol for wireless sensor network (WSN) applications in industry, control, home automation, health care, and smart grids, e.g., [1-4]. Many of these

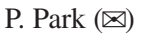

ACCESS Linnaeus Center, Electrical Engineering, Royal Institute of Technology,

Stockholm, Sweden

e-mail: pgpark@ee.kth.se.
} 


\section{Author's Proof}

applications require that packets ${ }^{1}$ are received with a given probability of success. In addition to such a reliability constraint, other applications ask for timely packet delivery [5]. It is known that IEEE 802.15.4 may have poor performance in terms of power consumption, reliability and delay [6], unless the MAC parameters are properly selected. It follows that (a) it is essential to characterize the protocol performance limitations, and (b) to develop methods to tune the IEEE 802.15.4 MAC parameters to enhance the network lifetime and improve the quality of the service experienced by the applications running on top of the network.

This book chapter focuses on the modelling and optimization of the performance metrics (reliability, delay, power consumption) for IEEE 802.15.4 WSNs. This problem is specially appealing for many control and industrial applications [2,7]. We show that existing analytical studies of IEEE 802.15.4 MAC are not adequate to capture the real-world protocol behavior, when there are retry limits to send packets, acknowledgements (ACKs), and unsaturated traffic. We derive and use a new model to pose an optimization problem, where the objective function is the power consumption of the nodes, and the constraints are the reliability and delay of the packet delivery. Our aim is the design of distributed and adaptive algorithms that are simple to implement on sensor nodes, but still flexible, scalable, and able to provide high quality of service for WSN applications.

The remainder of this chapter is as follows. Section 11.2 presents the overview of IEEE 802.15.4 standard. In Sect. 11.3, we summarize existing work on analytical modelling and adaptive tuning of the IEEE 802.15.4 MAC protocol. Section 11.4 presents the problem studied in the chapter. In Sect. 11.5, we propose a generalized Markov chain model of the carrier sense multiple access with collision avoidance (CSMA/CA) with retry limits and unsaturated traffic regime. In Sect. 11.6, the optimization problem to adapt the MAC parameters is investigated. In addition, practical issues on how to implement the algorithm on sensors are also discussed. Numerical results achieved during stationary and transitionary conditions are reported in Sect. 11.7. Finally, Sect. 11.8 concludes the chapter. An extended version of this chapter with further details is available as [8].

\subsection{Overview of the IEEE 802.15.4}

In this section, we give an overview of the key points of IEEE 802.15.4. The IEEE 802.15.4 standard specifies the physical layer and the MAC sublayer for low-rate Wireless Personal Area Networks (WPANs). The standard defines two channel access modalities: the beacon-enabled modality, which uses a slotted CSMA/CA and the optional Guaranteed Time Slot (GTS) allocation mechanism, and a simpler unslotted CSMA/CA without beacons. In the following, we focus on the

\footnotetext{
${ }^{1}$ Throughout this paper, we refer to packets as medium access control (MAC) protocol data units, or MAC frames.
} 


\section{Author's Proof}

11 Adaptive IEEE 802.15.4 Protocol for Control and Monitoring Applications

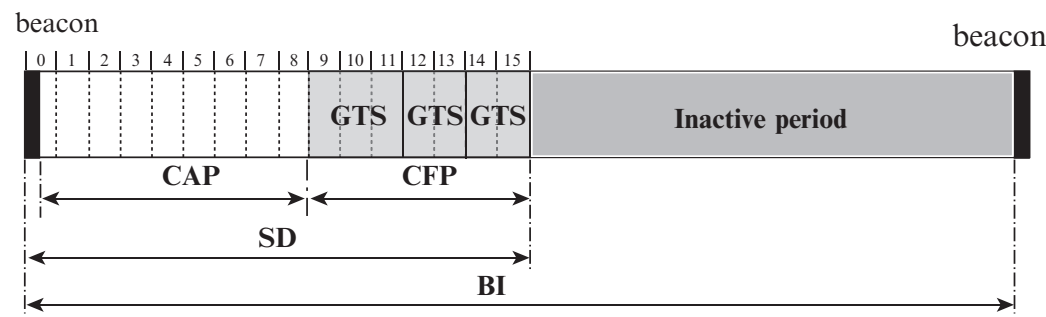

Fig. 11.1 Superframe structure of IEEE 802.15.4

beacon-enabled modality. Figure 11.1 shows a superframe structure of the beaconenabled mode. The coordinator periodically sends the beacon frames in every beacon interval (BI) to identify its personal area networks and to synchronize devices that communicate with it. The coordinator and devices can communicate during active period, called the superframe duration (SD), and enter the low-power mode during the inactive period. The structure of the superframe is defined by two parameters, the beacon order (BO) and the superframe order (SO), which determine the length of the superframe and its active period, respectively. The length of the superframe and the length of its active period are then defined as

$$
\begin{aligned}
& \mathrm{BI}=\text { aBaseSuperframeDuration } \times 2^{\mathrm{BO}}, \\
& \mathrm{SD}=\text { aBaseSuperframeDuration } \times 2^{\mathrm{SO}},
\end{aligned}
$$

where $0 \leq \mathrm{SO} \leq \mathrm{BO} \leq 14$ and aBaseSuperframeDuration is the number of symbols forming a superframe when SO is equal to 0 . In addition, the superframe is divided into 16 equally sized superframe slots of length aBaseSlotDuration. Each active period can be further divided into a Contention Access Period (CAP) and an optional Contention Free Period (CFP), composed of GTSs. A slotted CSMA/CA mechanism is used to access the channel of nontime critical data frames and GTS requests during the CAP. In the CFP, the dedicated bandwidth is used for time critical data frames. In the following section, we describe the data transmission mechanism for both CAP and CFP.

\subsubsection{CSMA/CA Mechanism of CAP}

Consider a node trying to transmit a data packet during CAP. In slotted CSMA/CA of IEEE 802.15.4, first the MAC sublayer initializes four variables, i.e., the number of backoffs $(\mathrm{NB}=0)$, contention window $(\mathrm{CW}=2)$, backoff exponent $(\mathrm{BE}=\operatorname{macMin} B E)$, and retransmission times $(\mathrm{RT}=0)$. Then the MAC sublayer delays for a random number of complete backoff periods in the range $\left[0,2^{\mathrm{BE}}-1\right]$ units. If the number of backoff periods is greater than the remaining number of backoff periods in the CAP, the MAC sublayer shall pause the backoff countdown 


\section{Author's Proof}

at the end of the CAP and resume it at the start of the CAP in the next superframe. Otherwise, the MAC sublayer counts its backoff delay. When the backoff period is zero, the node needs to perform the first clear channel assessment (CCA). The MAC sublayer proceeds if the remaining CSMA/CA algorithm steps (i.e., two CCAs), the frame transmission, and any acknowledgment can be completed before the end of the CAP. If the MAC sublayer cannot proceed, it shall wait until the start of the CAP in the next superframe and apply a further random backoff delay in the range $\left[0,2^{\mathrm{BE}}-1\right]$ units before evaluating whether it can proceed again. Otherwise the MAC sublayer proceeds the CCA in the current superframe. If two consecutive CCAs are idle, then the node commences the packet transmission. If either of the CCA fails due to busy channel, the MAC sublayer increases the value of both NB and BE by one, up to a maximum value macMaxCSMABackoffs and mac$M a x B E$, respectively. Hence, the values of NB and BE depend on the number of CCA failures of a packet. Once BE reaches $\operatorname{macMaxBE}$, it remains at the value macMaxBE until it is reset. If NB exceeds macMaxCSMABackoffs, then the packet is discarded due to channel access failure. Otherwise, the CSMA/CA algorithm generates a random number of complete backoff periods and repeats the process. Here, the variable macMaxCSMABackoffs represents the maximum number of times the CSMA/CA algorithm is required to backoff. If channel access is successful, the node starts transmitting packets and waits for ACK. The reception of the corresponding ACK is interpreted as successful packet transmission. If the node fails to receive ACK due to collision or ACK timeout, the variable RT is increased by one up to macMaxFrameRetries. If RT is less than macMaxFrameRetries, the MAC sublayer initializes two variables $\mathrm{CW}=0, \mathrm{BE}=$ macMinBE and follows the CSMA/CA mechanism to re-access the channel. Otherwise, the packet is discarded due to the retry limit. Note that the default MAC parameters are macMinBE $=3$, macMaxBE $=5$, macMaxCSMABackoffs $=4$, macMaxFrameRetries $=3$. See [1] for further details.

\subsubsection{GTS Allocation of CFP}

The coordinator is responsible for the GTS allocation and determines the length of the CFP in a superframe. To request the allocation of a new GTS, the device sends the GTS request command to the coordinator. The coordinator confirms its receipt by sending an acknowledgment frame within CAP. Upon receiving a GTS allocation request, the coordinator checks whether there are sufficient resources and, if possible, allocates the requested GTS. The GTS capacity in a superframe satisfies the following requirements:

1. The maximum number of GTSs to be allocated to devices is seven, provided there is sufficient capacity in the superframe.

2. The minimum length of a CAP is aMinCAPLength.

Therefore, the CFP length depends on the GTS requests and the currently available capacity in the superframe. 


\section{Author's Proof}

If there is sufficient bandwidth in the next superframe, the coordinator determines a device list for GTS allocation, based on a first-come-first-served (FCFS) policy. Then the coordinator includes the GTS descriptor, which is the device list that obtains GTSs in the following beacon to announce the allocation information. The coordinator makes this decision within aGTSDescPersistenceTime superframes. Note that on receipt of the acknowledgment to the GTS request command, the device continues to track beacons and wait for at most aGTSDescPersistenceTime superframes. A device uses the dedicated bandwidth to transmit the packet within the CFP. In addition, a transmitting device ensures that its transaction is complete one interframe spacing (IFS) period before the end of its GTS. See [1] for further details.

\subsection{Related Work}

We first discuss the literature concerning the analysis of IEEE 802.15.4, then we review previous work about adaptive MAC mechanisms for these protocols.

\subsubsection{Analytical Model of MAC}

In this section, we first discuss the literature concerning the analysis of CAP in IEEE 802.15.4, then we review previous work about the GTS allocation of CFP.

\subsubsection{Analytical model of CAP}

The modelling of CAP in IEEE 802.15.4 is related to IEEE 802.11 [9]. Both IEEE 802.11 and 802.15.4 are based on a MAC that uses a binary exponential backoff scheme. Bianchi's model describes the basic functionalities of the IEEE 802.11 through a Markov chain under saturated traffic and ideal channel conditions [10]. Extensions of this model have been used to analyze the packet reception rate [11], the delay [12], the MAC layer service time [13], and throughput [14] of IEEE 802.11.

The analysis of the packet delay, throughput, and power consumption of IEEE 802.15.4 WSNs has been the focus of several simulation-based studies, e.g., [15,16], and some more recent analytical works, e.g., [6]-[21]. Inspired by Bianchi's work, a Markov model for IEEE 802.15.4 and an extension with ACK mechanism have been proposed in [6] and [17]. A modified Markov model including retransmissions with finite retry limits has been studied in [18] as an attempt to model the CSMA/CA mechanism. However, the analysis gives inaccurate results because the power consumption and throughput expressions under unsaturated traffic with finite retry limits show a weak matching with simulation results. In [22], the authors consider finite retry limits with saturated traffic condition which is not a realistic scenario for WSN applications. 


\section{Author's Proof}

While the aforementioned works do not consider the active and inactive periods of the IEEE 802.15.4 nodes, this aspect is investigated in [19]-[21]. In [19], the authors assume unsaturated traffic and source nodes with a finite buffer, but only uplink communications. In [20], instead, downlink communications are also taken into account, but nodes are assumed to be equipped with infinite size buffers. Furthermore, in [20], the power consumption, reliability, and delay performance are not investigated. In [21], a throughput analysis has been performed by an extension of the Markov chain model proposed in [20]. The superframe structure, ACK, and retransmissions are considered. It is shown that the models in $[19,20]$, although very detailed, fail to couple with realistic simulation results. However, the proposed Markov chain does not model the length of data and ACK packets, which is crucial to analyze the performance metrics for IEEE 802.15.4 networks with low data rate. In [23], the authors approximate the CAP as the simple nonpersistent CSMA, which is a similar approach to [24]. However, the authors assume that the entire superframe duration is active, that is, $\mathrm{SO}=\mathrm{BO}$ without considering the inactive period.

The multihop network scenario is considered in [25]-[27]. In [25], the authors extended the framework proposed in [23] to 2-hop network scenarios, where sensors communicate with the coordinator through an intermediate relay node, which simply forwards the data packets received from the sources. In [26, 27], the authors propose the use of a relay for interconnecting two IEEE 802.15.4 clusters and analyze the performance using a queuing theory.

\subsubsection{Analytical Model of CFP}

Most of the literature does not consider satisfactorily the CFP, where the GTS mechanism operates. Simulation studies in [28]-[30] consider the CAP and CFP. An interesting theoretical performance evaluation of the GTS allocation has been proposed by Koubaa et al. [31]-[33] by using network calculus. These papers focus on the impact of the IEEE 802.15.4 standard parameters (SO, BO), the delay, throughput and energy consumption of GTS allocation. In [34], a round-robin scheduler is proposed to improve the bandwidth utilization based on a network calculus approach. Network calculus, however, assumes a continuous flow model (whereas communication happens through low data rate packets in reality) and it analyzes the worst-case of traffic flows (which leads to severe under-utilization of time slots in actual environments). Consequently, the difference between the network flow model of the network calculus approach and the actual behavior may be quite large. In [35], the authors analyze the stability, delay, and throughput of the GTS allocation mechanism based on the Markov chain model.

Some interesting algorithms have been proposed to improve the performance of GTS allocation mechanism. To maximize the bandwidth utilization, a smaller slot size and an offline message scheduling algorithm are proposed in [36] and [37], respectively. In [38], the delay constraint and bandwidth utilization are considered to design a GTS scheduling algorithm. Huang [39] proposes an adaptive GTS allocation scheme by considering low delay and fairness. 


\section{Author's Proof}

11 Adaptive IEEE 802.15.4 Protocol for Control and Monitoring Applications

\subsubsection{Adaptive Tuning of MAC}

Several algorithms to tune the MAC of IEEE 802.11 and IEEE 802.15.4 protocols have been proposed. The algorithms can be grouped in those based on the use of physical layer measurements, and those based on the use of link-layer information.

An adaptive tuning based on physical layer measurements has been investigated in $[24,40,41]$, where a $p$-persistent IEEE 802.11 protocol has been considered to optimize the average backoff window size. The channel access probability $p$ that maximizes the throughput or minimize the power consumption is derived. This algorithm and its scalability to the network size have been studied also for IEEE 802.15.4 [40]. However, that study was less successful, because the channel sensing mechanism, the optional ACK, and retransmission mechanisms are hard to be approximated by a $p$-persistent MAC. Furthermore, in [40] and [41] a saturated traffic regime is assumed, which is a scenario of little interest for typical WSN applications.

Link-based optimizations for IEEE 802.11 and 802.15.4 have been investigated in [42]-[46], where simple window adjustment mechanisms that are based on ACK transmissions have been considered. In these papers, the algorithms adapt the contention window size depending on the successful packet transmission, packet collision and channel sensing state, but the algorithms are not grounded on an analytical study. In [42], different backoff algorithms are presented to improve the channel throughput and the fairness of channel usage for IEEE 802.11. A fair backoff algorithm is studied also in [43] and [44]. A link-based algorithm of the IEEE 802.15.4 random backoff mechanism to maximize the throughput has been presented in [45]. In [46], a dynamic tuning algorithm of the contention window size is evaluated on goodput, reliability, and average delay.

An IEEE 802.15.4 enhancement based on the use of link-layer information has some drawback. First, it requires a modification of the standard. Then, although linkbased mechanisms are simple to implement, the ACK mechanism may be costly since it introduces large overhead for small packets. For instance, alarm messages in an industrial application are a single byte, whereas the ACK has a size of 11 bytes. In addition, the ACK mechanism requires extra waiting time. Moreover, link-based algorithms adapt the MAC parameters for each received ACK, which mean a slow and inefficient adaptation to network, traffic, and channel variations.

\subsection{Problem Formulation}

We consider a star network with a coordinator, and $N$ nodes transmitting to the coordinator. These nodes use the beacon-enabled slotted CSMA/CA and ACK. The important parameters of the CSMA/CA algorithm for our study are the minimum value of the backoff exponent macMinBE, the maximum number of backoffs macMaxCSMABackoffs and the maximum number of retries macMaxFrameRetries that each node can select. See details of IEEE 802.15.4 in [1] and [47]. 


\section{Author's Proof}

In this book chapter, we propose a novel modelling and adaptive tuning of IEEE 802.15.4 for reliable and timely communication while minimizing the energy consumption. The protocol is adjusted dynamically by a constrained optimization problem that each node of the network solves. The objective function, denoted by $\widetilde{E}_{\text {tot }}$, is the total energy consumption for transmitting and receiving packets of a node. The constraints are given by the probability of successful packet delivery (reliability) and average delay. The constrained optimization problem for a generic transmitting node in the network is

$$
\begin{array}{cl}
\min _{\mathbf{V}} & \widetilde{E}_{\text {tot }}(\mathbf{V}) \\
\text { s.t. } & \widetilde{R}(\mathbf{V}) \geq R_{\min }, \\
& \widetilde{D}(\mathbf{V}) \leq D_{\max }, \\
& \mathbf{V}_{0} \leq \mathbf{V} \leq \mathbf{V}_{m} .
\end{array}
$$

The decision variables of the node $\mathbf{V}=\left(m_{0}, m, n\right)$ are

$$
\begin{aligned}
m_{0} & \triangleq \text { macMinBE }, \\
m & \triangleq \text { macMaxCSMABackoffs }, \\
n & \triangleq \text { macMaxFrameRetries } .
\end{aligned}
$$

$\widetilde{R}(\mathbf{V})$ is the reliability, and $R_{\min }$ is the minimum desired probability for successful packet delivery. $\widetilde{D}(\mathbf{V})$ is the average delay for a successfully received packet, and $D_{\text {max }}$ is the desired maximum average delay. The constraint $\mathbf{V}_{0} \leq \mathbf{V} \leq \mathbf{V}_{m}$ captures the limited range of the MAC parameters. In the problem, we used the symbol ${ }^{\sim}$ to evidence that the energy, reliability, and delay expression are approximations. The corresponding variables without denote the true values. We will show later that we use approximations of high accuracy and reduced computational complexity so that nodes can solve the problem.

Main contributions of this chapter are the following: (a) the modelling of the relation between the MAC parameters of IEEE 802.15.4 and the selected performance metrics, (b) the derivation of simple relations to characterize the operations of the MAC by computationally affordable algorithms, (c) formulation and solution of a novel optimization problem for the MAC parameters, (d) discussion on a practical implementation of the optimization by an adaptive algorithm and (e) performance evaluations of the algorithm by simulation of both stationary and transient network conditions.

Unlike previous work, we propose a generalized Markov model of the exponential backoff process including retry limits, ACKs, and unsaturated traffic regime. However, the numerical evaluation of these performance metrics asks, in general, for heavy computations. This is a drawback when using them to optimize the IEEE 802.15.4 MAC parameters by in-network processing [48] because a complex computation is out of reach for resource-limited sensing devices. Therefore, we devise a simplified and effective method that reduces drastically the computational complexity while ensuring a satisfactory accuracy. 


\section{Author's Proof}

Based on our novel modelling, we propose an adaptive tuning of MAC parameters that uses the physical layer measurement of channel sensing. This adaptive IEEE 802.15.4 MAC protocol is furnished with two distinctive features: it does not require any modification of the existing standard, and it makes an optimization of all the MAC parameters of IEEE 802.15.4. Specifically, in contrast to link-based adaptation [42]-[46], our algorithm does not require ACK mechanism or request to send/clear to send (RTS/CTS) handshakes (or related standard modifications). In contrast to [24]-[41], we do not use the (inaccurate) $p$-persistent approximation and the modification of the standard therein proposed, and we do not require any hardware modification to make an estimate of the signal-to-noise ratio. Our adaptive tuning optimizes the considered MAC parameters, all at once, and not only some of them, as proposed in [24]-[46].

The proposed adaptive IEEE 802.15.4 MAC improves the power efficiency substantially while guaranteeing reliability and delay constraints. The adaptation is achieved by distributed asynchronous iterations that only require channel condition information, the number of nodes of the network, and the traffic load. We show that the convergence is fast and robust to errors in the estimation of the channel condition, number of nodes, and traffic load. A good fairness is also achieved.

\subsection{Analytical Modelling of IEEE 802.15.4}

In a star network, all $N$ nodes contend to send data to the PAN coordinator, which is the data sink. We assume no hidden node. Throughout this paper, we consider applications where nodes asynchronously generate packets with probability $1-q$, when a node sends a packet successfully, discards a packet, or the sampling interval expires. Otherwise, a node stays for $L_{0} S_{b}$ s without generating packets with probability $q$, where $L_{0}$ is an integer and $S_{b}$ is the time unit aUnitBackoffPeriod (corresponding to 20 symbols). The data packet transmission is successful if an ACK packet is received. In addition, we assume that the channel is sensed busy or idle without errors.

In such a scenario, we propose an effective analytical model of the slotted CSMA/CA by a Markov chain. The chain enables us to derive the objective function, energy (11.3a), and constraints on reliability (11.3b) and delay (11.3c) of the optimization problem.

\subsubsection{Markov Chain Model}

In this section, we develop a generalized Markov chain model of the slotted CSMA/CA mechanism of beacon-enabled IEEE 802.15.4. Compared to previous results, e.g., [6,17]-[21], the novelty of this chain consists in the modelling of the retry limits for each packet transmission, ACK, the inclusion of unsaturated traffic regimes, and packet size. 


\section{Author's Proof}

Let $s(t), c(t)$ and $r(t)$ be the stochastic processes representing the backoff stage, the state of the backoff counter and the state of retransmission counter at time $t=$ $0,1, \ldots \infty$ experienced by a node to transmit a packet. By assuming independent probability that nodes start sensing, the stationary probability $\tau$ that a node attempts a first carrier sensing in a randomly chosen slot time is constant and independent of other nodes. The triple $(s(t), c(t), r(t))$ defines the three-dimensional Markov chain in Fig. 11.2, where we use $(i, k, j)$ to denote its state. We denote the MAC parameters by $\mathbf{V}=\left(m_{0}, m, n\right), m_{b} \triangleq \operatorname{macMaxBE}, W_{0} \triangleq 2^{m_{0}}, W_{m} \triangleq 2^{\min \left(m_{0}+m, m_{b}\right)}$.

The Markov chain consists of four main parts corresponding to the idle-queue states, backoff states, CCA states, and packet transmission states. The states

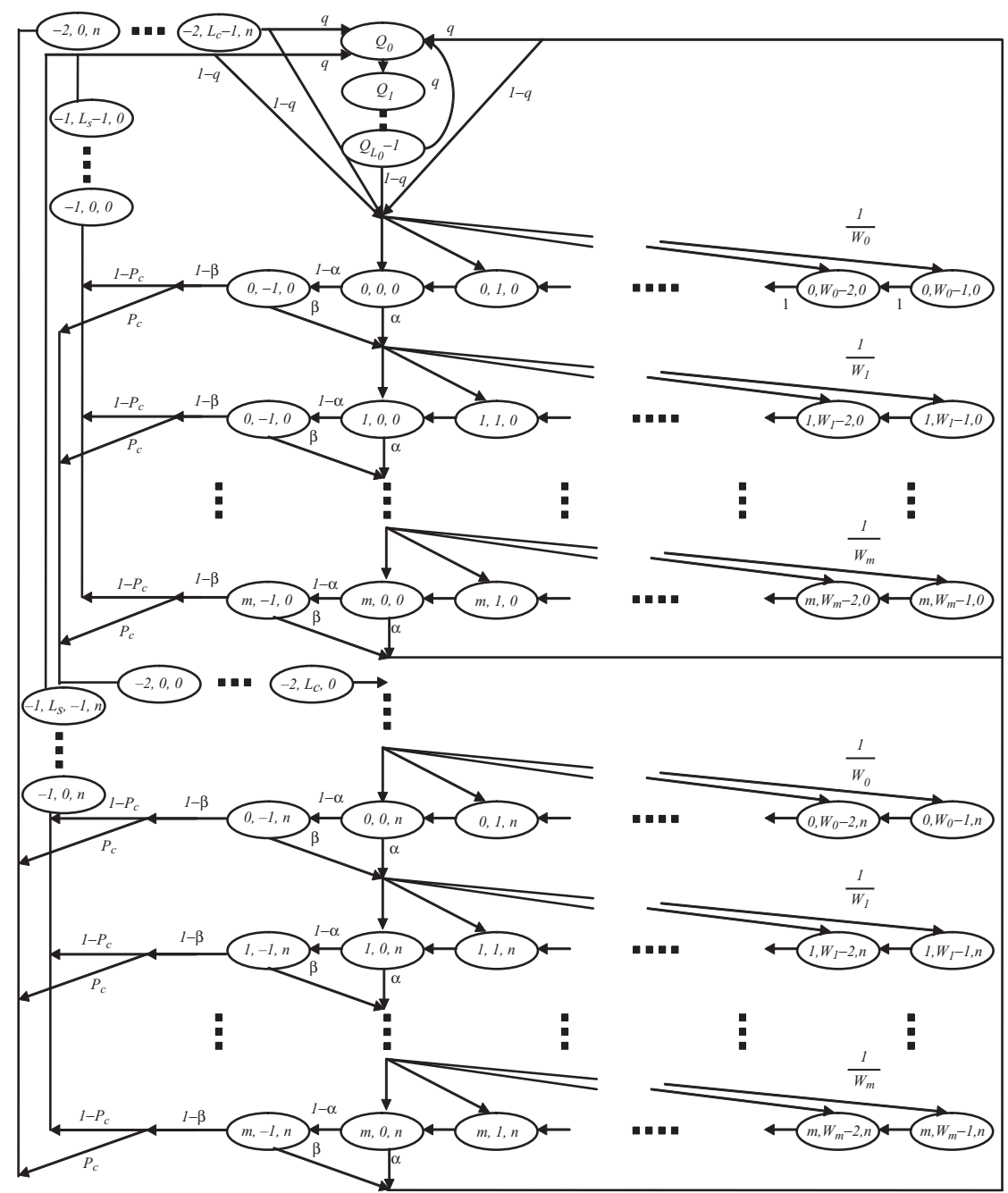

Fig. 11.2 Markov chain model for CSMA/CA of IEEE 802.15.4 


\section{Author's Proof}

$\left(Q_{0}, \ldots, Q_{L_{0}-1}\right)$ in the top of Fig. 11.2 correspond to the idle-queue states when the packet queue is empty and the node is waiting for the next packet generation time. Note that these states take into account the sampling interval. The states $\left(i, W_{m}-1, j\right), \ldots,\left(i, W_{0}-1, j\right)$ represent the backoff states. The states $(i, 0, j)$ and $(i,-1, j)$ represent first CCA $\left(\mathrm{CCA}_{1}\right)$ and second $\mathrm{CCA}\left(\mathrm{CCA}_{2}\right)$, respectively. Let $\alpha$ be the probability that $\mathrm{CCA}_{1}$ is busy, and $\beta$ the probability that $\mathrm{CCA}_{2}$ is busy. The states $(-1, k, j)$ and $(-2, k, j)$ correspond to successful transmission and packet collision, respectively. By knowing the duration of an ACK frame, ACK timeout, IFS, data packet length, and header duration, we define the time duration of a successful packet transmission $L_{\mathrm{s}}$ and the corresponding time for packet collision $L_{\mathrm{c}}$ as

$$
\begin{aligned}
& L_{\mathrm{s}}=L+t_{\mathrm{ack}}+L_{\mathrm{ack}}+\mathrm{IFS}, \\
& L_{\mathrm{c}}=L+t_{\mathrm{m}, \mathrm{ack}},
\end{aligned}
$$

where $L$ is the total length of a packet including overhead and payload, $t_{\text {ack }}$ is ACK waiting time, $L_{\text {ack }}$ is the length of the ACK frame, and $t_{\mathrm{m} \text {,ack }}$ is the timeout length of the ACK, see details in [1].

We have the following result on how to compute the stationary probability of the Markov chain:

Lemma 1. Let the stationary probability of the Markov chain in Fig. 11.2 be denoted

$$
b_{i, k, j}=\lim _{t \rightarrow \infty} P(s(t)=i, c(t)=k, r(t)=j),
$$

where $i \in(-2, m), k \in\left(-1, \max \left(W_{i}-1, L_{s}-1, L_{c}-1\right)\right), j \in(0, n)$. Then, for $0 \leq i \leq m$

$$
b_{i, k, j}=\frac{W_{i}-k}{W_{i}} b_{i, 0, j}, \quad 0 \leq k \leq W_{i}-1,
$$

where

$$
W_{i}= \begin{cases}2^{i} W_{0}, & 0 \leq i \leq m_{b}-m_{0} \\ 2^{m_{b}}, & \text { otherwise }\end{cases}
$$

and

$$
b_{i, 0, j}=\left[(1-\alpha)(1-\beta) P_{\mathrm{c}} \sum_{i=0}^{m}(\alpha+(1-\alpha) \beta)^{i}\right]^{j}(\alpha+(1-\alpha) \beta)^{i} b_{0,0,0},
$$




\section{Author's Proof}

282

P. Park et al.

with

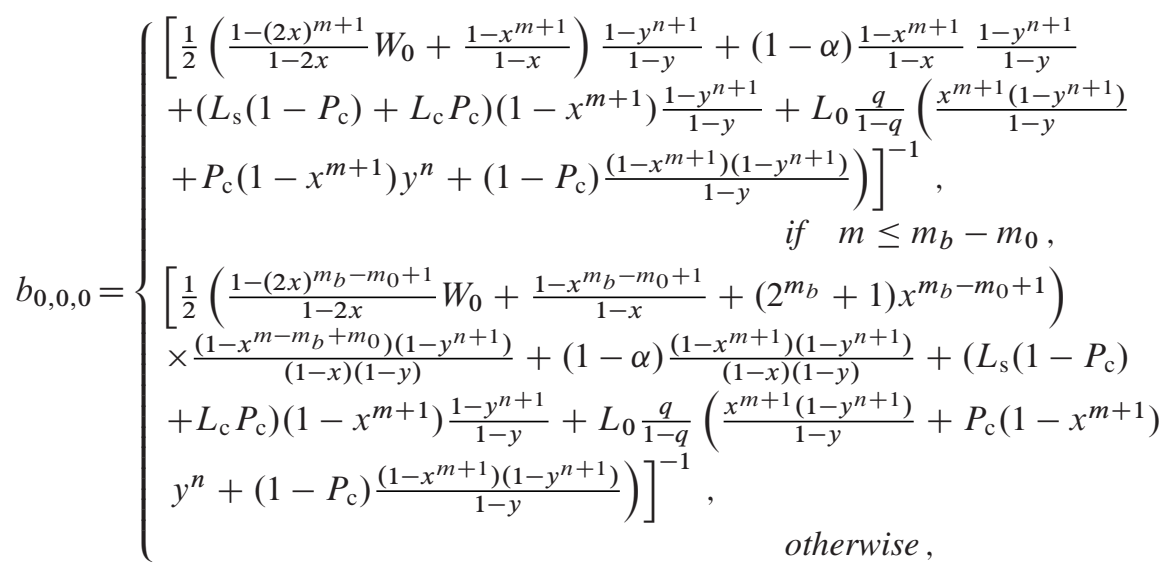

where $x=\alpha+(1-\alpha) \beta, y=P_{\mathrm{c}}\left(1-x^{m+1}\right)$, and $P_{c}$ is the collision probability. Moreover,

$$
b_{-1, k, j}=\left(1-P_{\mathrm{c}}\right)(1-x) \sum_{i=1}^{m} b_{i, 0, j}, \quad 0 \leq k \leq L_{\mathrm{s}}-1,
$$

and

$$
b_{-2, k, j}=P_{\mathrm{c}}(1-x) \sum_{i=1}^{m} b_{i, 0, j}, \quad 0 \leq k \leq L_{\mathrm{c}}-1 .
$$

Proof. A proof is given in [8].

We remark here that the stationary probability $b_{0,0,0}$, which plays a key role in the analysis below, is different from the corresponding term in $[6,17]-[21]$ due to our more detailed modelling of the retransmissions, ACK, unsaturated traffic, and packet size. In the following section, we demonstrate the validity of the Markov chain model by Monte Carlo simulations.

Now, starting from Lemma 1, we derive the channel sensing probability $\tau$ and the busy channel probabilities $\alpha$ and $\beta$. The probability $\tau$ that a node attempts $\mathrm{CCA}_{1}$ in a randomly chosen time slot is

$$
\tau=\sum_{i=0}^{m} \sum_{j=0}^{n} b_{i, 0, j}=\frac{1-x^{m+1}}{1-x} \frac{1-y^{n+1}}{1-y} b_{0,0,0} .
$$

This probability depends on the probability $P_{\mathrm{c}}$ that a transmitted packet encounters a collision, and the probabilities $\alpha$ and $\beta$. These probabilities are derived in the following. 


\section{Author's Proof}

11 Adaptive IEEE 802.15.4 Protocol for Control and Monitoring Applications

The term $P_{\mathrm{c}}$ is the probability that at least one of the $N-1$ other nodes transmits in the same time slot. If all nodes transmit with probability $\tau$, we have

$$
P_{\mathrm{c}}=1-(1-\tau)^{N-1}
$$

Similar to [6], we derive the busy channel probabilities $\alpha$ and $\beta$ as follows. We have

$$
\alpha=\alpha_{1}+\alpha_{2}
$$

where $\alpha_{1}$ is the probability of finding channel busy during $\mathrm{CCA}_{1}$ due to data transmission by one of the other $N-1$ nodes, namely,

$$
\alpha_{1}=L\left(1-(1-\tau)^{N-1}\right)(1-\alpha)(1-\beta),
$$

and $\alpha_{2}$ is the probability of finding the channel busy during $\mathrm{CCA}_{1}$ due to ACK transmission:

$$
\alpha_{2}=L_{\text {ack }} \frac{N \tau(1-\tau)^{N-1}}{1-(1-\tau)^{N}}\left(1-(1-\tau)^{N-1}\right)(1-\alpha)(1-\beta),
$$

where $L_{\text {ack }}$ is the length of the ACK. In a similar way, the probability of finding the channel busy during $\mathrm{CCA}_{2}$ is

$$
\beta=\frac{1-(1-\tau)^{N-1}+N \tau(1-\tau)^{N-1}}{2-(1-\tau)^{N}+N \tau(1-\tau)^{N-1}} .
$$

Now, we are in the position to derive the carrier sensing probability $\tau$ and the busy channel probabilities $\alpha$ and $\beta$ by solving the system of nonlinear equations (11.10)-(11.12), see details in [49]. From these probabilities, one can derive the expressions for the reliability, delay for successful packet delivery, and power consumption in (11.3). Unfortunately, there is no closed-form expression for these probabilities, but the system of (11.10)-(11.12) must be solved by numerical methods. This may be computationally demanding and therefore inadequate for use in embedded sensor devices. In the following, we therefore instead present a simplified analytical model of the reliability, delay for successful packet delivery, and power consumption. The key idea is that sensor nodes can estimate the busy channel probabilities $\alpha$ and $\beta$ and the channel sensing probability $\tau$. Therefore, nodes can exploit local measurements to evaluate the performance metrics, rather than solving complex nonlinear equations. Details follow in the sequel, where we derive these approximate expressions for (11.3a)-(11.3c).

\subsubsection{Reliability}

The main contributions of this section are a precise and approximated expression of the reliability (11.3b) of the optimization problem (11.3), where we recall the reliability is the probability of successful packet reception. 


\section{Author's Proof}

Proposition 1. The reliability is

$$
R(\boldsymbol{V})=1-\frac{x^{m+1}\left(1-y^{n+1}\right)}{1-y}-y^{n+1},
$$

where $x$ and $y$ are given in Lemma 1 .

Proof. A proof is given in [8].

Approximation 1. An approximation of the reliability is

$$
\widetilde{R}(\boldsymbol{V})=1-x^{m+1}(1+\widetilde{y})-\widetilde{y}^{n+1}
$$

where

$$
\begin{aligned}
& \widetilde{y}=\left(1-\left(1-(1+x)(1+\hat{y}) \widetilde{b}_{0,0,0}\right)^{N-1}\right)\left(1-x^{2}\right), \\
& \widetilde{b}_{0,0,0}=2\left[W_{0}(1+2 x)(1+\hat{y})+2 L_{\mathrm{s}}\left(1-x^{2}\right)(1+\hat{y})\right. \\
&\left.+L_{0} q /(1-q)\left(1+\hat{y}^{2}+\hat{y}^{n+1}\right)\right]^{-1},
\end{aligned}
$$

and $\hat{y}=\left(1-(1-\tau)^{N-1}\right)\left(1-x^{2}\right)$.

Proof. A proof is given in [8].

We remark that $\widetilde{R}(\mathbf{V})$ is a function of the measurable busy channel probabilities $\alpha$ and $\beta$, the channel access probability $\tau$ and the MAC parameters $m_{0}, m_{b}, m, n$. The approximation is based on estimated values of $x$ and $\tau$. Monte Carlo simulations in [8] validate the approximated model of the reliability.

\subsubsection{Delay}

In this section, we derive the constraint of the average delay (11.3c). The average delay for a successfully received packet is defined as the time interval from the instant the packet is at the head of its MAC queue and ready to be transmitted, until the transmission is successful and the ACK is received. In this section, we develop an approximation for such an average delay, which is given by Approximation 2. To this aim, we need some intermediate technical steps. In particular, we characterize (a) the expression of the delay for a successful transmission at time $j+1$ after $j$ th events of unsuccessful transmission due to collision and (b) the expected value of the approximated backoff delay due to busy channel.

Let $D_{j}$ be the random time associated with the successful transmission of a packet at the $j$ th backoff stage. Denote with $\mathcal{A}_{j}$ the event of a successful transmission at time $j+1$ after $j$ th events of unsuccessful transmission. Let $\mathcal{A}^{\text {tot }}$ be the event of successful transmission within the total attempts $n$. Then, the delay for a successful transmission after $j$ th unsuccessful attempts is 


\section{Author's Proof}

11 Adaptive IEEE 802.15.4 Protocol for Control and Monitoring Applications

$$
D=\sum_{j=0}^{n} \mathbb{1}_{\mathcal{A}_{j} \mid \mathcal{A}^{\mathrm{tot}} D_{j},}
$$

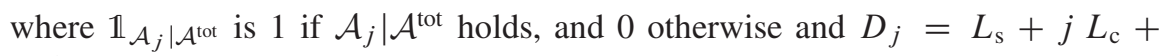
$\sum_{h=0}^{j} T_{h}$, with $T_{h}$ being the backoff stage delay, $L_{\mathrm{s}}$ is the packet successful transmission time, and $L_{\mathrm{c}}$ is the packet collision transmission time as defined in (11.4).

Lemma 2. The probability of successful transmission at time $j+1$ after jth events of unsuccessful transmission due to collision is

$$
\operatorname{Pr}\left(\mathcal{A}_{j} \mid \mathcal{A}^{\mathrm{tot}}\right)=\frac{(1-y) y^{j}}{1-y^{n+1}} .
$$

Proof. A proof is given in [8].

In the following, we derive the total backoff delay $T_{h}$. Let $T_{h, i}$ be the random time needed to obtain two successful CCAs from the selected backoff counter value at backoff level $i$. Recall that a node transmits the packet when the backoff counter is 0 and two successful CCAs are detected [1]. Denote with $\mathcal{B}_{i}$ the event occurring when the channel is busy for $i$ times, and then idle at time $i+1$. Let $\mathcal{B}^{\text {tot }}$ be the event of having a successful sensing within the total number of $m$ sensing attempts. If the node accesses an idle channel after its $i$ th busy CCA, then

$$
T_{h}=\sum_{i=0}^{m} \mathbb{1}_{\mathcal{B}_{i} \mid \mathcal{B}^{\text {tot }}} T_{h, i},
$$

where

$$
T_{h, i}=2 T_{s c}+\sum_{k=1}^{i} T_{h, k}^{s c}+\sum_{k=0}^{i} T_{h, k}^{b}
$$

and $2 T_{s c}$ is the successful sensing time, $\sum_{k=1}^{i} T_{h, k}^{s c}$ is the unsuccessful sensing time due to busy channel during CCA, and $\sum_{k=0}^{i} T_{h, k}^{b}$ is the backoff time. The expected value of the approximated backoff delay is

$$
\begin{aligned}
\mathbb{E}\left[\widetilde{T}_{h}\right]=2 S_{b}(1 & +\frac{1}{4}\left(\frac{1-\gamma}{1-\gamma^{m+1}}\left(2 W_{0} \frac{1-(2 \gamma)^{m+1}}{1-2 \gamma}-\frac{3(m+1) \gamma^{m+1}}{1-\gamma}\right)\right. \\
& \left.\left.+\frac{3 \gamma}{1-\gamma}-\left(W_{0}+1\right)\right)\right)
\end{aligned}
$$

where $\gamma=\max (\alpha,(1-\alpha) \beta)$. A proof is given in [8].

Now, we are in the position to derive an approximation of the average delay for successfully received packets. 


\section{Author's Proof}

286

P. Park et al.

Approximation 2. The expected value of the approximated delay is

$$
\widetilde{D}(\boldsymbol{V})=T_{s}+\mathbb{E}\left[\widetilde{T}_{h}\right]+\left(\frac{y}{1-y}-\frac{(n+1) y^{n+1}}{1-y^{n+1}}\right)\left(T_{c}+\mathbb{E}\left[\widetilde{T}_{h}\right]\right)
$$

Proof. A proof is given in [8].

Validation of the approximated model of the average packet delay is given in [8].

\subsubsection{Power Consumption}

Here, we derive the objective function, power consumption of the node (11.3a) of the optimization problem (11.3). We propose two models for the average power consumption, depending on the radio state during the backoff mechanism specified by the IEEE 802.15.4 standard. Let us denote by I-mode and S-mode the situation when the radio is set in idle mode or in sleep mode during backoff period, respectively.

Approximation 3. The approximated energy consumption of the I-mode $\widetilde{E}_{\text {tot,i }}(\boldsymbol{V})$ is

$$
\begin{aligned}
\widetilde{E}_{\mathrm{tot}, \mathrm{i}}(\boldsymbol{V})= & \frac{P_{\mathrm{i}} \tau}{2}\left[\frac{(1-x)\left(1-(2 x)^{m+1}\right)}{(1-2 x)\left(1-x^{m+1}\right)} W_{0}-1\right]+P_{\mathrm{sc}}(2-\alpha) \tau+(1-\alpha) \\
& \times(1-\beta) \tau\left(P_{\mathrm{t}} L+P_{\mathrm{i}}+L_{\mathrm{ack}}\left(P_{\mathrm{r}}\left(1-P_{\mathrm{c}}\right)+P_{\mathrm{i}} P_{\mathrm{c}}\right)\right)+P_{\mathrm{w}} q \\
& \times\left(x^{m+1}(1+y)+P_{\mathrm{c}}\left(1-x^{2}\right) y^{n}+\left(1-P_{\mathrm{c}}\right)\left(1-x^{2}\right)(1+y)\right) \widetilde{b}_{0,0,0}
\end{aligned}
$$

and of the S-mode is

$$
\begin{aligned}
\widetilde{E}_{\mathrm{tot}, \mathrm{s}}(\boldsymbol{V})= & P_{\mathrm{sc}}(2-\alpha) \tau+(1-\alpha)(1-\beta) \tau\left(P_{\mathrm{t}} L+P_{\mathrm{i}}+L_{\mathrm{ack}}\left(P_{\mathrm{r}}\left(1-P_{\mathrm{c}}\right)\right.\right. \\
& \left.\left.+P_{\mathrm{i}} P_{\mathrm{c}}\right)\right)+P_{\mathrm{w}}\left(\tau-\frac{\widetilde{b}_{0,0,0}\left(1-(0.5 x)^{m+1}\right)\left(1-y^{n+1}\right)}{W_{0}(1-0.5 x)(1-y)}\right)
\end{aligned}
$$

where

$$
\widetilde{b}_{0,0,0} \approx \frac{2}{W_{0} r_{1}+2 r_{2}}
$$

with

$$
\begin{aligned}
r_{1} & =(1+2 x)(1+\hat{y}), \\
r_{2} & =L_{\mathrm{s}}\left(1-x^{2}\right)(1+\hat{y})+K_{0}\left(1+\hat{y}^{2}+\hat{y}^{n+1}\right), \\
\hat{y} & =\left(1-(1-\tau)^{N-1}\right)\left(1-x^{2}\right) .
\end{aligned}
$$




\section{Author's Proof}

11 Adaptive IEEE 802.15.4 Protocol for Control and Monitoring Applications

$P_{\mathrm{i}}, P_{\mathrm{sc}}, P_{\mathrm{sp}}, P_{\mathrm{w}}, P_{\mathrm{t}}$, and $P_{\mathrm{r}}$ are the average power consumption in idle-listen, channel sensing, sleep, wake-up, transmit, and receive states, respectively.

Proof. A proof is given in [8].

Monte Carlo simulations validate also this approximated model in [8].

\subsection{IEEE 802.15.4 Optimization}

In the previous sections, we developed the expressions of the performance metrics. Here, we present a novel approach where each node locally solves an optimization problem. Consider the reliability, delay, and power consumption as investigated in Sect. 11.5. The optimization problem (11.3) can be defined by using (11.14) of Approximation 1 for the reliability constraint, (11.18) of Approximation 2 for the delay constraint and (11.19) or (11.20) of Approximation 3 for the power consumption. Note that the power consumption is given by (11.19) if the I-mode is selected, and it is given by (11.20), if the $S$-mode is selected. The solution of the optimization problem gives the optimal MAC parameters $\left(m_{0}, m, n\right)$ that each node uses to minimize its energy expenditure, subject to reliability and delay constraints. Notice that the problem is combinatorial because the decision variables take on discrete values.

A vector of decision variables $\mathbf{V}$ is feasible if the reliability and delay constraints are satisfied. The optimal solution may be obtained by checking every combination of the elements of $\mathbf{V}$ that gives feasibility, and then checking the combination that gives the minimum objective function. Clearly, this approach may have a high computational complexity, since there are $6 \times 4 \times 8=192$ combinations of MAC parameters to check [1]. Therefore, in the following we propose an algorithm that gives the optimal solution by checking a reduced number of combinations.

We remark here that the reliability and power consumption of both I-mode and $S$-mode are increasing functions of $n$, see details in [8]. This property is quite useful to solve (11.3) by a simple algorithm with reduced computational complexity, as we see next.

The search of optimal MAC parameters uses an iterative procedure according to the component-based method [50]. In particular, the probabilities $\alpha, \beta$, and $\tau$ are estimated periodically by each node. If a node detects a change of these probabilities, then the node solves the local optimization problem (11.3) using these new estimated values. The solution is achieved by finding the value of $n$ that minimizes the energy consumption given a pair of values for $m_{0}$ and $m$. Since the power consumption is increasing with $n$, it follows that the minimum is attained at the lowest value of $n$ that satisfies the constraints. Given that the reliability is increasing with $n$, simple algebraic passages give that such a value is $n=f\left(m_{0}, m\right)$, with

$$
f\left(m_{0}, m\right)=\left\lceil\frac{\ln \left(1-x^{m+1}(1+\widetilde{y})-R_{\min }\right)}{\ln (\widetilde{y})}-1\right\rceil,
$$




\section{Author's Proof}

where $\widetilde{y}=\left(1-(1-\widetilde{\tau})^{N-1}\right)\left(1-x^{2}\right)$ and

$$
\widetilde{\tau}=\frac{2 r_{3}}{2^{m_{0}} r_{1}+2 r_{2}},
$$

with

$$
\begin{aligned}
& r_{1}=(1+2 x)(1+\hat{y}), \\
& r_{2}=L_{\mathrm{s}}\left(1-x^{2}\right)(1+\hat{y})+\frac{L_{0} q\left(1+\hat{y}^{2}+\hat{y}^{n+1}\right)}{1-q}, \\
& r_{3}=(1+x)(1+\hat{y}),
\end{aligned}
$$

and $\hat{y}=\left(1-(1-\tau)^{N-1}\right)\left(1-x^{2}\right)$. Equation (11.22) returns the optimal retry limits given a pair $m_{0}, m$. Notice that $x$ and $\hat{y}$ are measurable since the node estimates $\alpha, \beta$, and $\tau$. By using this simple algorithm, a node checks just $6 \times 4=24$ combinations of the MAC parameters $m_{0}, m$ instead of $6 \times 4 \times 8=192$ combinations, which would be required by an exhaustive search.

We have seen by the Approximations 1, 2 and 3 that the performance metrics are a function of the busy channel probabilities $\alpha$ and $\beta$ and the channel access probability $\tau$. Once these probabilities are known at a node, the optimal MAC parameters of that node can be readily computed by the proposed algorithm. In the algorithm, the number of nodes and packet generation rates are assumed to be known, whereas the busy channel probability and channel access probability are periodically estimated during the sensing states of the MAC layer, and they do not require an ACK mechanism. The robustness of the algorithm to possible errors in the estimation of the number of nodes and traffic load is investigated in Sect. 11.7.3.

The average busy channel probabilities $\alpha$ and $\beta$ are estimated at each node while sending a data packet to the coordinator. These probabilities are initialized at the beginning of the node's operation. The estimations of the busy channel probabilities and the channel access probability use a first-order filter. When the node senses the channel at $\mathrm{CCA}_{1}$ or $\mathrm{CCA}_{2}$, these probabilities are updated using the following recursions

$$
\begin{aligned}
& \alpha_{k+1}=\delta_{b} \alpha_{k}+\left(1-\delta_{b}\right) \hat{\alpha}_{k}, \\
& \beta_{k+1}=\delta_{b} \beta_{k}+\left(1-\delta_{b}\right) \hat{\beta}_{k},
\end{aligned}
$$

where $k$ denotes the update step for some $\delta_{b} \in(0,1)$, respectively. Note that $\hat{\alpha}$ and $\hat{\beta}$ are the busy channel probability measurements of $\mathrm{CCA}_{1}$ and $\mathrm{CCA}_{2}$, respectively. Each node only counts the number of busy channel events during $\mathrm{CCA}_{1}$ and $\mathrm{CCA}_{2}$ state to obtain $\hat{\alpha}$ and $\hat{\beta}$, respectively. Therefore, a node does not require any extra communication and sensing state to estimate these probabilities compared to the IEEE 802.15.4 standard. By contrast, the estimation algorithms for IEEE 802.11 proposed in [24] and [51] are not energy efficient since a node needs to sense the channel state during the backoff stage. This allows one to estimate the average 


\section{Author's Proof}

length of the idle period. Hence, these schemes are implementable only in I-mode. By contrast, our scheme is applied in both I-mode and S-mode and does not require any computation load during the backoff stage. An analysis of the impact of parameter estimation errors is investigated in Sect. 11.7.3.

During an initialization phase of the algorithm, each node uses the initial MAC parameters $m_{0}=3, m_{b}=8, m=4, n=3$. The busy channel probabilities $\alpha$ and $\beta$ and the channel access probability $\tau$ are estimated during the channel sensing state of IEEE 802.15.4 without any extra states. The application requirements are communicated by the coordinator to the node if there are changes. It is also possible that each node makes a decision of application requirements depending on the data type, such as strict delay requirements for alarm messages.

\subsection{Numerical Results}

In the following, we present Monte Carlo simulations to analyze the performance of our adaptive tuning algorithm of the MAC parameters, under both stationary and transient conditions. The analytical modelling that we have proposed in Sect. 11.5 is based on a Markov chain that has been validated experimentally in [52]. The Monte Carlo simulations that we use here are representative of the real-world behavior of the network.

In the stationary conditions, the application requirements and network scenario are constant, whereas in transient condition there are variations. The simulations are based on the specifications of the IEEE 802.15.4 and the practical implementation aspects described in Sect. 11.6. In the simulations, the network considers the $I$-mode and $S$-mode of the node to compare the performance on the reliability, average packet delay, and power consumption. Furthermore, we investigate the fairness of resource allocation, robustness to network changes, and sensitivity to inaccurate parameter estimations. Note that it is hard to compare our algorithm to other algorithms from the literature as the link-based ones [42-46], because they modify the IEEE 802.15.4 standard and are focused on different performance metrics (e.g., throughput). However, it is possible to show that our algorithm outperform significantly the results in [42-46], due to that these results use the ACK feedback, which has a low update frequency with respect to the channel and network variations, whereas our algorithm reacts much faster. Details follow in the sequel.

\subsubsection{Protocol Behavior in Stationary Conditions}

In this subsection, we are interested in the improvement of performance metrics of the proposed scheme at stationary conditions of the network, namely without changing application requirements and network scenarios. We also present a fairness analysis of the adaptive protocol. Simulation data was collected out of 5 runs, each lasting $2 \times 10^{5}$ time slots. 


\section{Author's Proof}

Figure 11.3 compares the reliability, average delay, and power gain values of the protocol as obtained by our algorithm and with default MAC parameters. Both the I-mode and $S$-mode for various traffic configurations and requirements are considered. The requirements for both the I-mode and $S$-mode are $R_{\min }=0.9,0.95, D_{\max }=50$, and $R_{\min }=0.95, D_{\max }=20,100 \mathrm{~ms}$, respectively. Figure 11.3 a shows that both $I$-mode and $S$-mode satisfy the reliability constraint for different traffic regimes. We observe strong dependence of the reliability of the default MAC protocol with different traffic regime due to the fixed MAC parameters. At the high traffic regime $q=0.2$, the reliability of default MAC is 0.86 . In Fig. 11.3b, the delay constraint is fulfilled in both $I$-mode and $S$-mode. Observe that the average delay in the $I$-mode decreases when traffic regime is low $q \geq 0.5$. This is due to that the optimal MAC parameters at higher traffic regime increase more than the ones at lower traffic regime to satisfy the reliability constraint.

Recall that the target of our proposed adaptive algorithm is to use the tradeoff between application constraints and energy consumption instead of just maximization of reliability or minimization of delay. Therefore, to characterize quantitatively the power consumption, we define the power gain as

$$
\rho=\frac{E_{\mathrm{def}}-E_{\mathrm{tot}}(\mathbf{V})}{E_{\mathrm{def}}}
$$

where $E_{\text {def }}$ and $E_{\text {tot }}(\mathbf{V})$ are the average power consumption of $I$-mode or $S$-mode for default MAC and proposed scheme, respectively. The closer $\rho$ is to 1 , the better power efficiency. Figure 11.3c shows that the power gain increases as traffic load increases. This improvement is higher for $S$-mode than I-mode, e.g., power gain $\rho \approx 0.49$ for $S$-mode with $R_{\min }=0.95, D_{\max }=100$. Although there is a strong dependence of the power gain on the traffic regime, our proposed algorithm gives a better energy efficiency than the default MAC. Therefore, the numerical results show clearly the effectiveness of our adaptive IEEE 802.15.4 MAC protocol while guaranteeing the constraints.

Next, we observe the tradeoff between the power consumption, reliability, and delay constraints. Figure 11.4 shows the dependence of the power consumption in $S$-mode with reliability and delay constraints for a given traffic load, packet length, and number of nodes. Observe that as the delay constraint becomes strict the power consumption increases. In other words, the reliability constraint of $S$-mode is less critical than delay constraint, see more results in [8].

The fairness of resource management is one of the most important concerns when implementing the tuning algorithm of the MAC parameters. We use Jain's fairness index [53] to show the fairness of our proposed scheme for both I-mode and S-mode. We compute the fairness index of 10 nodes in a stable network. The closer fairness index to 1 , the better the achieved fairness. Figure 11.5 shows the fairness index of the reliability for the different requirements and traffic configurations with a given length of the packet and number of nodes. Figure 11.5 reports a very high fairness achievement on reliability greater than 0.999 . A similar behavior is found for delay 


\section{Author's Proof}

11 Adaptive IEEE 802.15.4 Protocol for Control and Monitoring Applications

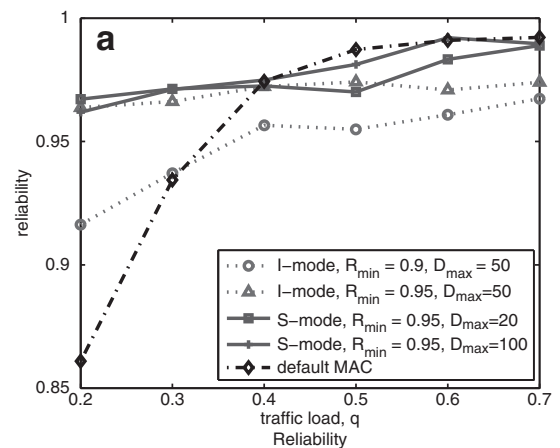

Reliability

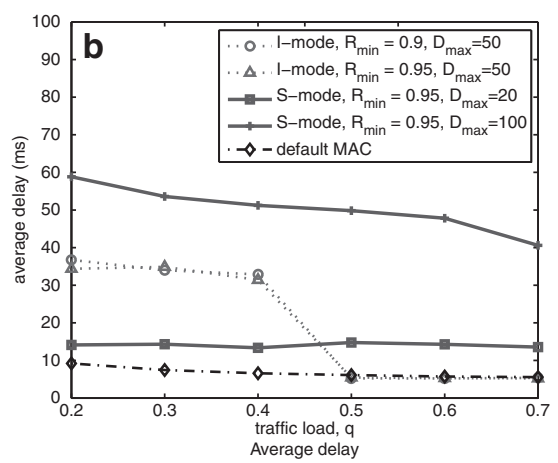

Average delay

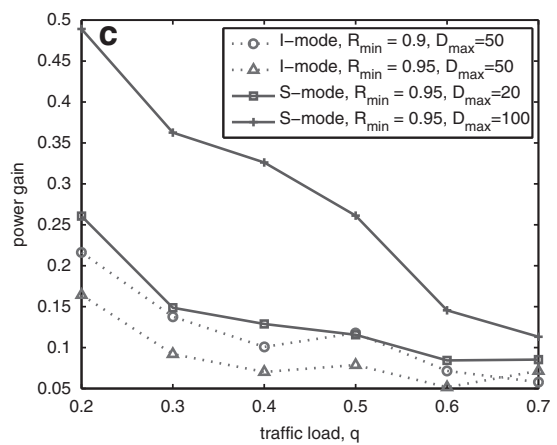

Power gain

Fig. 11.3 Stationary condition: reliability, average delay, and power gain of the I-mode, $S$ mode of proposed scheme and IEEE 802.15.4 with default parameter (macMinBE $=3$, macMaxBE $=5$, macMaxCSMABackoffs $=4$, macMaxFrameRetries $=3$ ) as a function of the traffic load $q=0.2, \ldots, 0.7$, the reliability requirement $R_{\min }=0.9,0.95$ and delay requirement $D_{\max }=20,50,100 \mathrm{~ms}$ for the length of the packet $L=7$ and $N=10$ nodes. Note that "default MAC" refers to IEEE 802.15.4 with default MAC parameters 


\section{Author's Proof}

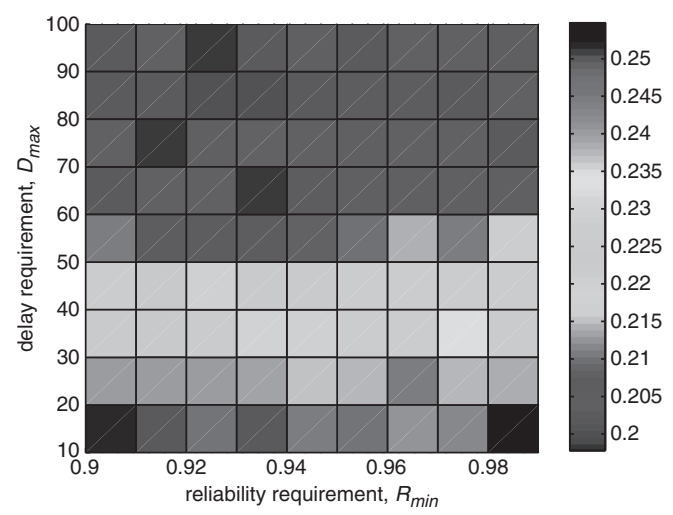

Fig. 11.4 Stationary network condition: power consumption of $S$-mode as a function of reliability constraint $R_{\min }=0.9, \ldots, 0.99$ and delay requirement $D_{\max }=10, \ldots, 100 \mathrm{~ms}$ for the traffic load $q=0.5$, the length of packet $L=3$ and $N=10$ nodes

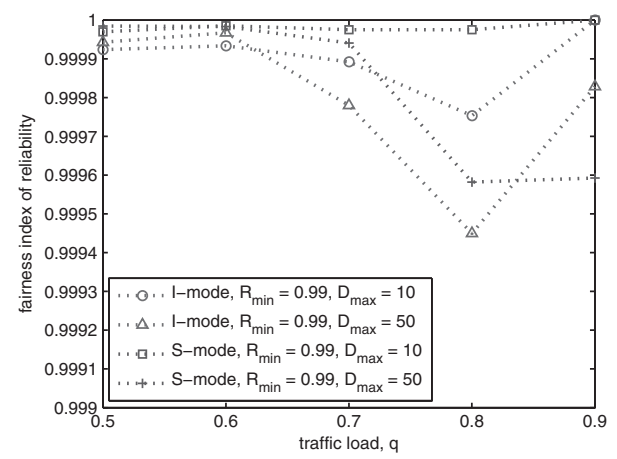

Fig. 11.5 Fairness index of the reliability as a function of the traffic load $q=0.5, \ldots, 0.9$, reliability requirement $R_{\min }=0.99$, and delay requirement $D_{\max }=10,50 \mathrm{~ms}$ for the length of the packet $L=3$ and $N=10$ nodes

and power consumption. In other words, the MAC parameters of each node converge to the optimal MAC parameter values. Therefore, we conclude that most of the nodes can equally share the common medium.

\subsubsection{Protocol Behavior in Transient Conditions}

The adaptive IEEE 802.15.4 MAC protocol is based on the estimation of the busy channel probabilities $\alpha$ and $\beta$ and the channel access probability $\tau$. In this section, we investigate the convergence time of the optimal MAC parameters obtained by our adaptive algorithm when the delay constraint changes. 


\section{Author's Proof}

Figure 11.6 shows the behavior of channel state, MAC parameters, reliability, and packet delay when the delay requirement changes for both I-mode and S-mode with a given traffic load, length of packets, and number of nodes. Figure 11.6a reports the busy channel probabilities $\alpha$ and $\beta$ and channel access probability $\tau$ over time. In Sect. 11.6, we noticed that the update frequency of $\alpha, \beta, \tau$ is different. $\tau$ is updated in each aUnitBackoffPer-iod and $\alpha$ and $\beta$ are updated when a node stays in CCA $_{1}$ and $\mathrm{CCA}_{2}$. Hence, the update order of $\alpha, \beta$, and $\tau$ is $\tau$ first, then $\alpha$, and finally $\beta$. We remark here that the update frequency of link-based adaptation is lower than the update frequency of $\beta$ of our algorithm since link-based adaptation requires an ACK transmission [42]-[46]. The update frequency of channel estimation is a critical issue where the traffic load is low, such as in monitoring applications.

Figure 11.6b shows the adaptation of the MAC parameters. The optimal $\left(m_{0}, m, n\right)$ of $I$-mode and $S$-mode adapts to $(3,2,0)$ and $(8,5,0)$, respectively, before the requirement changes. Observe that the algorithm returns different parameters for I-mode and S-mode due to the different power consumption model, see details in Sect. 11.5. After the requirement changes at time $26 \mathrm{~s}$, the MAC parameters $\left(m_{0}, m, n\right)$ of $S$-mode adapt from $(8,5,0)$ to $(5,2,0)$. We observe that the convergence of the MAC parameters is very fast since our algorithm is based on an analytical model instead of heuristic considerations as in link-based adaptation, where the algorithms adapt the contention window size by the ACK transmission [42]-[46]. In addition, recall that our adaptive IEEE 802.15.4 MAC is based on the physical sensing information before transmitting packets.

Figures 11.6c shows the cumulative packet reception rate of I-mode and S-mode. Note that the oscillation of reliability is due to packet loss. In Fig. 11.6c, the reliability of $S$-mode is larger than I-mode since the MAC parameters $m_{0}$ and $m$ are larger than the ones of I-mode before the requirement changes. By the same argument, we observe that the packet delay of $S$-mode is about six times the one measured of $I$-mode in Fig. 11.6d. In addition, the packet delay is much more variable in $S$-mode than the one in I-mode. Specifically, with I-mode, we have a reduction in the average MAC delay and a shorter tail for the MAC delay distribution with respect to the $S$-mode. After the requirement changes, the packet delay converges to around $10 \mathrm{~ms}$. In addition, the reliability decreases due to the decreasing of the parameters $m_{0}$ and $m$ in Fig. 11.6c.

\subsubsection{Robustness and Sensitivity Analysis}

The performance analysis carried out so far assumed that the number of nodes and traffic configuration are fixed. This assumption has allowed us to verify the effectiveness of our adaptive algorithm for IEEE 802.15.4 MAC in steady-state conditions. However, one of the critical issues in the design of wireless networks is that of time-varying conditions. Therefore, in the following analysis, we will investigate how our algorithm reacts to changes in the number of nodes and traffic load when each node has an erroneous estimation of these parameters. 


\section{Author's Proof}
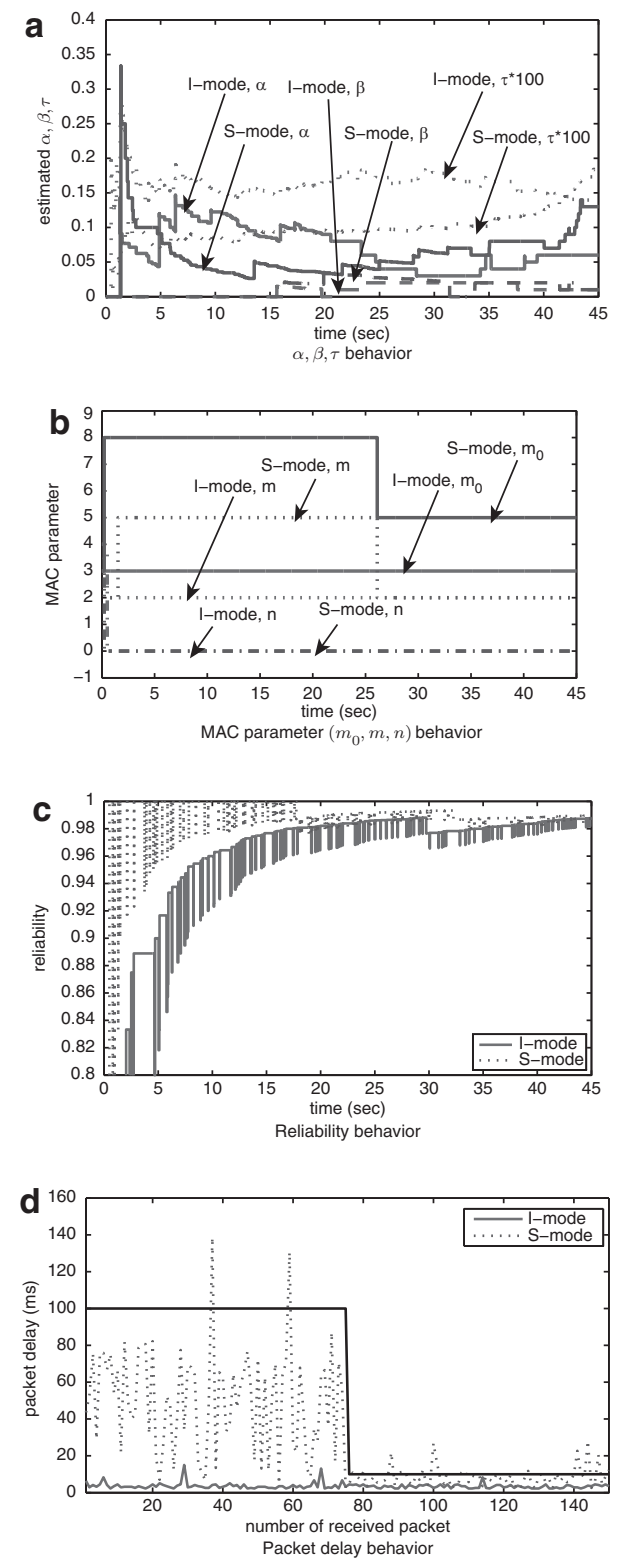

Fig. 11.6 Transient condition: busy channel probabilities, channel access probability, MAC parameters, reliability and delay of $I$-mode and $S$-mode for the traffic load $q=0.6$, length of the packet $L=3$ and $N=10$ nodes when the delay requirement changes from $D_{\max }=100 \mathrm{~ms}$ to $D_{\max }=10 \mathrm{~ms}$ at $26 \mathrm{~s}$ 


\section{Author's Proof}

11 Adaptive IEEE 802.15.4 Protocol for Control and Monitoring Applications

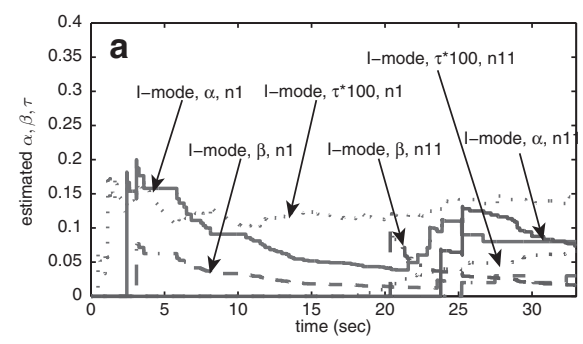

$\alpha, \beta, \tau$ behavior

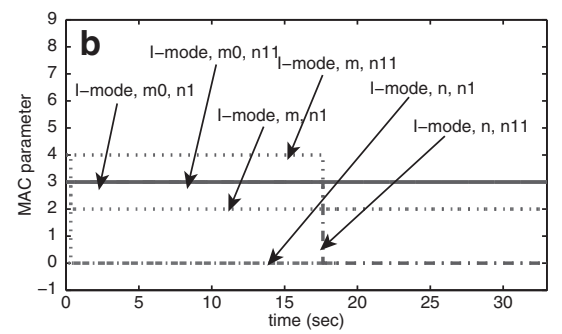

MAC parameter $\left(m_{0}, m, n\right)$ behavior

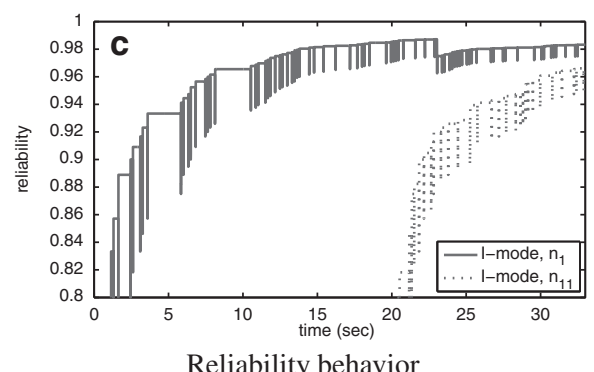

Fig. 11.7 Robustness when the number of nodes changes: busy channel probabilities, channel access probability, MAC parameters, and reliability behavior of I-mode when the number of nodes changes sharply from $N=10$ to $N=20$ at time $17.6 \mathrm{~s}$. Note that $n_{1}$ and $n_{11}$ represent the behavior of one of $N=10$ nodes plus new nodes after time 17.6s. Traffic load is $q=0.6$, length of the packet is $L=3$, and the reliability and delay constraint are $R_{\min }=0.95$ and $D_{\max }=100 \mathrm{~ms}$, respectively

Figure 11.7 shows the dynamical behavior of nodes using the I-mode when the number of nodes changes from $N=10$ to $N=20$ with an erroneous estimation of the number of nodes. At time $17.6 \mathrm{~s}$, the number of nodes sharply increases to 20 , when it was estimated to be 10 . We assume that the wrong estimation happens due to some errors in the estimation phase or a biasing induced by the hidden-node phenomenon. This causes a significant increase of the contention level. Note that $n_{1}$ is one of the existing nodes before the network change and $n_{11}$ is one of the new nodes that enters the network at time $17.6 \mathrm{~s}$ using its initial MAC parameters. In Fig. 11.7a, we observe that the busy channel and channel access probabilities of 


\section{Author's Proof}

node $n_{11}$ become stable after the network changes by updating the MAC parameters. Fig. $11.7 \mathrm{~b}$ shows that the MAC parameters $\left(m_{0}, m, n\right)$ converge to $(3,2,0)$ for nodes $n_{1}$ and $n_{11}$. The figures indicate that the system reacts correctly to the erroneous estimation of the number of nodes after a few seconds. In Fig. 11.7c, the reliability fulfills the requirement $R_{\min }=0.95$ for both the existing and new nodes. Similar behaviors are observed for $S$-mode, see further details in [8].

Figure 11.8 presents the behavior of the node when the traffic load changes sharply from $q=0.8$ to $q=0.5$ at time $25.6 \mathrm{~s}$. Nodes use a wrong estimation of the traffic load, which is estimated to be $q=0.8$, after the traffic load changes. The results indicate that our algorithm is quite effective for the traffic configuration
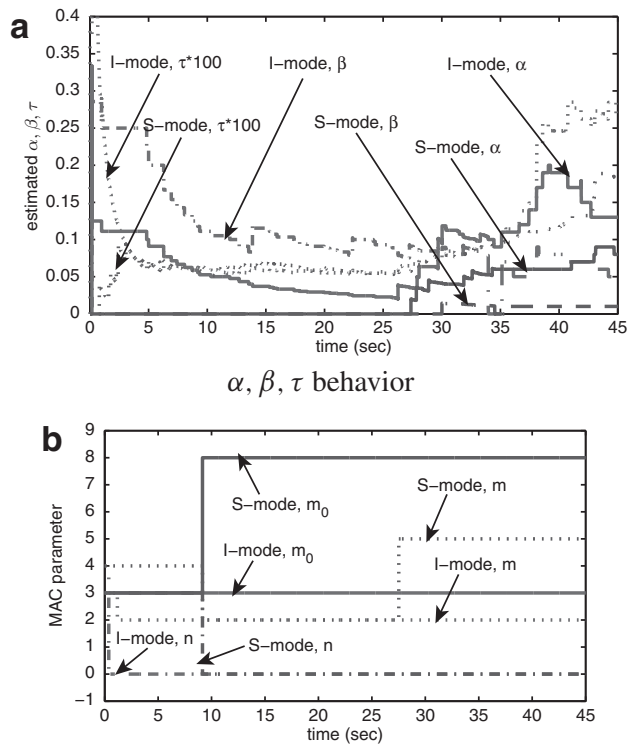

MAC parameter $\left(m_{0}, m, n\right)$ behavior

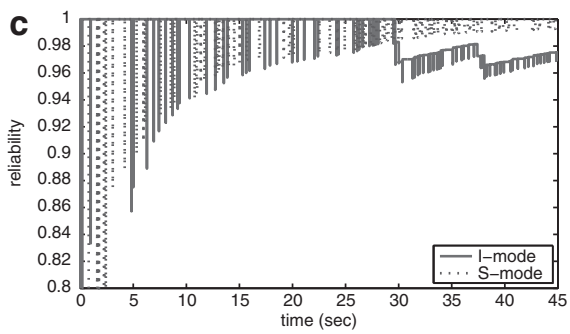

Reliability behavior

Fig. 11.8 Robustness when the traffic load changes: busy channel probabilities, channel access probability, MAC parameters, reliability and delay behavior of I-mode and S-mode when the traffic load changes sharply from $q=0.8$ to $q=0.5$ at time $25.6 \mathrm{~s}$. The length of the packet is $L=3$ and the reliability and delay constraint are $R_{\min }=0.95$ and $D_{\max }=100 \mathrm{~ms}$, respectively 


\section{Author's Proof}

change. In Fig. 11.8a, the busy channel and channel access probability increase as a result of higher traffic regime $q=0.5$ for both $I$-mode and $S$-mode. Figure $11.8 \mathrm{~b}$ shows that the parameter $m$ of $S$-mode updates from 2 to 5 due to the increasing busy channel probability after the traffic load changes at time $28 \mathrm{~s}$. The figure indicates that the system reacts correctly to the erroneous estimation of traffic configuration and, in a few seconds, the estimation of $\alpha, \beta$, and $\tau$ makes it possible to reach the optimal MAC parameters. In Fig. $11.8 \mathrm{c}$, the reliability requirement $R_{\min }=0.95$ is fulfilled for both I-mode and $S$-mode. The reliability of I-mode is greater than 0.95 with some fluctuations after the traffic load increases.

In Sect. 11.5, we assume that the ideal channel sensing capability of hardware without hidden node terminals. However, this assumption may not be practical due to the hardware failure and time-varying wireless condition. Hence, it is important analyze the sensitivity of our adaptive IEEE 802.15.4 MAC to the estimation errors. Figure 11.9 illustrates the sensitivity of the proposed scheme with respect to the estimation errors to the busy channel probabilities $\alpha$ and $\beta$ and the channel access probability $\tau$. The normalized root mean squared deviation (NRMSD) between the optimal MAC parameters with exact estimation and the ones with erroneous estimation is used as the indicator of sensitivity. The normalization is taken over the range of MAC parameters $\left(m_{0}, m, n\right)$. The NRMSD is approximately below $10 \%$ if the percentage of error is smaller than $20 \%$ for $\alpha, \beta$, and $\tau$. It is interesting to observe that $m_{0}$ of $I$-mode is very robust to errors. This is due to the power consumption model, i.e., to the dominant factor $m_{0}$ of power consumption in I-mode. The robustness of MAC parameter is $m_{0}>n>m$ and $n>m>m_{0}$ for $I$-mode and $S$-mode, respectively. We can show that errors below $20 \%$ in the estimation of $\alpha, \beta, \tau$ give a performance degradation below $3 \%$ in terms of reliability, packet delay and energy gain for low traffic load.

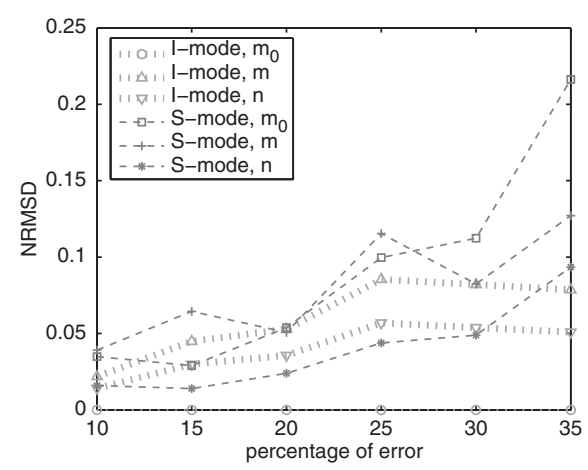

Fig. 11.9 Sensitivity: NRMSD of $I$-mode and S-mode when the traffic load $q=0.6$, length of the packet $L=3$, reliability requirement $R_{\min }=0.95$ and delay requirement $D_{\max }=100 \mathrm{~ms}$, and $N=10$ nodes with different percentage error in busy channel probabilities $\alpha$ and $\beta$ and channel access probability $\tau$ 


\section{Author's Proof}

\subsection{Conclusions}

In this chapter, we developed an analysis based on a generalized Markov chain model of IEEE 802.15.4, including retry limits, ACKs, and unsaturated traffic regime. Then, we presented an adaptive MAC algorithm for minimizing the power consumption while guaranteeing reliability and delay constraints of the IEEE 802.15.4 protocol. The algorithm does not require any modifications of the standard. The adaptive algorithm is grounded on an optimization problem, where the objective function is the total power consumption, subject to constraints of reliability and delay of the packet delivery, and the decision variables are the MAC parameters (macMinBE, macMaxCSMABackoffs, macMaxFrameRetries) of the standard. The proposed adaptive MAC algorithm is easily implementable on sensor nodes by estimating the busy channel and channel access probability.

We investigated the performance of our algorithm under both stationary and transient conditions. Numerical results showed that the proposed scheme is efficient and ensures a longer lifetime of the network. In addition, we showed that, even if the number of active nodes, traffic configuration, and application constraints change sharply, our algorithm allows the system to recover quickly and operate at its optimal parameter, by estimating just the busy channel and channel access probabilities. We also studied the robustness of the protocol to possible errors during the estimation process on number of nodes and traffic load. Results indicated that the protocol reacts promptly to erroneous estimations.

Acknowledgements This work was supported by the EU project FeedNetBack, the Swedish Research Council, the Swedish Strategic Research Foundation, and the Swedish Governmental Agency for Innovation Systems. A preliminary version of this book chapter has been presented at ACM/IEEE International Conference on Information Processing in Sensor Networks, Stockholm, Sweden, 2010, and has been submitted to a journal.

\section{References}

1. IEEE Std 802.15.4-2996, September, Part 15.4: Wireless Medium Access Control (MAC) and Physical Layer (PHY) Specifications for Low-Rate Wireless Personal Area Networks (WPANs), IEEE, 2006. [Online]. Available: http://www.ieee802.org/15.

2. A. Willig, K. Matheus, and A. Wolisz, "Wireless technology in industrial networks," Proceedings of the IEEE, pp. 1130-1151, June 2005.

3. A. Speranzon, C. Fischione, K. H. Johansson, and A. Sangiovanni-Vincentelli, "A distributed minimum variance estimator for sensor networks," IEEE Journal on Selected Areas in Communications, pp. 609-621, May 2008.

4. C. Fischione, A. Speranzon, K. H. Johansson, and A. Sangiovanni-Vincentelli, "Peer-to-peer estimation over wireless sensor networks via Lipschitz optimization," in ACM/IEEE IPSN, 2009.

5. T. Abdelzaher, T. He, and J. Stankovic, "Feedback control of data aggregation in sensor networks," in IEEE CDC, 2004.

6. S. Pollin, M. Ergen, S. C. Ergen, B. Bougard, F. Catthoor, A. Bahai, and P. Varaiya, "Performance analysis of slotted carrier sense IEEE 802.15.4 acknowledged uplink transmissions," in IEEE WCNC, 2008. 


\section{Author's Proof}

11 Adaptive IEEE 802.15.4 Protocol for Control and Monitoring Applications

7. J. R. Moyne and D. M. Tilbury, "The emergence of industrial control networks for manufacturing control, diagnostics, and safety data," Proceedings of the IEEE, pp. 29 - 47, Jan. 2007.

8. P. Park, P. D. Marco, C. Fischione, and K. H. Johansson, "Adaptive IEEE 802.15.4 protocol for reliable and timely communications," KTH, Tech. Rep., 2009, submitted for journal publication.

9. IEEE Std 802.11 Wireless LAN Medium Access Control (MAC) and Physical Layer (PHY) Specifications, IEEE, 1999. [Online]. Available: http://www.ieee802.org/11.

10. G. Bianchi, "Performance analysis of the IEEE 802.11 distributed cordination function," IEEE Journal on Selected Areas in Communications, pp. 535 - 547, March 2000.

11. P. Chatzimisios, A. C. Boucouvalas, and V. Vitsas, "IEEE 802.11 packet delay - A finite retry limit analysis," in IEEE GLOBECOM, 2003.

12. Z. Hadzi-Velkov and B. Spasenovski, "Saturation throughput-delay analysis of IEEE 802.11 in fading channel," in IEEE ICC, 2003.

13. O. Tickoo and B. Sikdar, "Queueing analysis and delay mitigation in IEEE 802.11 random access MAC based wireless networks," in IEEE INFOCOM, 2004.

14. H. Wu, Y. Peng, K. Long, S. Cheng, and J. Ma, "Performance of reliable transport protocol over IEEE 802.11 wireless LAN: Analysis and enhancement," in IEEE INFOCOM, 2002.

15. J. Zheng and M. L. Lee, "A comprehensive performance study of IEEE 802.15.4," in IEEE Press Book, 2004.

16. A. Koubaa, M. Alves, and E. Tovar, "A comprehensive simulation study of slotted CSMA/CA for IEEE 802.15.4 wireless sensor networks," in IEEE IWFCS, 2006.

17. S. Pollin, M. Ergen, S. C. Ergen, B. Bougard, L. V. D. Perre, F. Catthoor, I. Moerman, A. Bahai, and P. Varaiya, "Performance analysis of slotted carrier sense IEEE 802.15.4 medium access layer," in IEEE GLOBECOM, 2006.

18. P. K. Sahoo and J. P. Sheu, "Modeling IEEE 802.15.4 based wireless sensor network with packet retry limits," in ACM PE-WASUN, 2008.

19. J. Mišió and V. Mišió, "Access delay for nodes with finite buffers in ieee 802.15 .4 beacon enabled pan with uplink transmissions," Computer Communications, pp. 1152-1166, April 2005.

20. J. Mišió, S. Shaf, and V. Mišió, "Performance of a beacon enabled IEEE 802.15.4 cluster with downlink and uplink traffic," IEEE Transactions Parallel and Distributed Systems, pp. 361376, April 2006.

21. C. Y. Jung, H. Y. Hwang, D. K. Sung, and G. U. Hwang, "Enhanced markov chain model and throughput analysis of the slotted CSMA/CA for IEEE 802.15.4 under unsaturated traffic conditions," IEEE Transactions on Vehicular Technology, pp. 473-478, Jan. 2009.

22. Z. Tao, S. Panwar, G. Daqing, and J. Zhang, "Performance analysis and a proposed improvement for the ieee 802.15.4 contention access period," in IEEE WCNC, 2006.

23. I. Ramachandran, A. K. Das, and S. Roy, "Analysis of the contention access period of ieee 802.15.4 mac," ACM Transactions on Sensor Networks, pp. 641-651, 2007.

24. F. Cali, M. Conti, and E. Gregori, "IEEE 802.11 protocol: design and performance evaluation of an adaptive backoff mechanism," IEEE Journal on Selected Areas in Communications, pp. $1774-1786$, Sept. 2000.

25. M. Martalo, S. Busanelli, and G. Ferrari, "Markov chain-based performance analysis of multihop ieee 802.15.4 wireless networks," Performance Evaluation, pp. 722-741, 2009.

26. J. Mišsió, J. Fung, and V. Mišió, "Interconnecting 802.15.4 clusters in master-slave mode: queueing theoretic analysis," in IEEE ISPAN, 2005.

27. J. Mišió and R. Udayshankar, "Slave-slave bridging in 802.15 .4 beacon enabled networks," in IEEE WCNC, 2007.

28. G. Lu, B. Krishnamachari, and C. Raghavendra, "Performance evaluation of the IEEE 802.15.4 MAC for low-rate low-power wireless networks," in IEEE IPCCC, 2004.

29. N. Timmons and W. Scanlon, "Analysis of the performance of IEEE 802.15.4 for medical sensor body area networking," in IEEE SECON, 2004.

30. L. Changle, L. Huan-Bang, and R. Kohno, "Performance evaluation of ieee 802.15.4 for wireless body area network (wban)," in IEEE ICC Workshops, 2009. 


\section{Author's Proof}

31. A. Koubaa and Y. Q. Song, "Evaluation and improvement of response time bounds for realtime applications under non-preemptive fixed priority scheduling," International Journal of Production and Research (IJPR), pp. 2899-2913, July 2004.

32. A. Koubaa, M. Alves, and E. Tovar, "GTS allocation analysis in IEEE 802.15.4 for real-time wireless sensor networks," in IEEE IPDPS, 2006, pp. 25-29.

33. — "Energy and delay trade-off of the GTS allocation mechanism in IEEE 802.15.4 for wireless sensor networks," International Journal of Communication Systems, 2006.

34. A. Koubaa, M. Alves, and E. Tovar", "i-GAME: An implicit GTS allocation mechanism in IEEE 802.15.4 for time-sensitive wireless sensor networks," in Proc. 18th Euromicro Conf. Real-Time Systems (ECRTS 06), 2006.

35. P. Park, C. Fischione, and K. H. Johansson, "Performance analysis of GTS allocation in beacon enabled IEEE 802.15.4," in IEEE SECON, 2009.

36. L. Cheng, X. Zhang, and A. G. Bourgeois, "GTS allocation scheme revisited," Electronics Letters, pp. 1005-1006, 2007.

37. S. E. Yoo, D. Y. Kim, M. L. Pham, Y. M. Doh, E. C. Choi, and J. D. Huh, "Scheduling support for guaranteed time services in IEEE 802.15.4 low rate WPAN," in IEEE RTCSA, 2005.

38. C. Na, Y. Yang, and A. Mishra, "An optimal GTS scheduling algorithm for time-sensitive transactions in IEEE 802.15.4 networks," Computer Networks, pp. 2543-2557, Sept 2008.

39. Y. Huang, A. Pang, and H. Hung, "An adaptive GTS allocation scheme for IEEE 802.15.4," IEEE Transactions Parallel Distrib. Syst., pp. 641-651, 2008.

40. K. Yedavalli and B. Krishnamachari, "Enhancement of the IEEE 802.15.4 MAC protocol for scalable data collection in dense sensor networks," in ICST WiOPT, 2008.

41. R. Bruno, M. Conti, and E. Gregori, "Optimization of efficiency and energy consumption in p-persistent CSMA-based wireless LANs," IEEE Transactions on Mobile Computing, pp. 10 - 31, Jan. 2002.

42. Q. Pang, S. C. Liew, J. Y. B. Lee, and V. C. M. Leung, "Performance evaluation of an adaptive backoff scheme for WLAN: Research articles," Wirel. Commun. Mob. Comput., pp. 867-879, Dec. 2004.

43. V. Bharghavan, A. J. Demers, S. Shenker, and L. Zhang, "MACAW: A media access protocol for wireless LAN's," ACM SIGCOMM, 1994.

44. B. Bensaou, Y. Wang, and C. C. Ko, "Fair medium access in 802.11 based wireless ad-hoc networks," in ACM MobiHoc, 2000.

45. J. G. Ko, Y. H. Cho, and H. Kim, "Performance evaluation of IEEE 802.15.4 MAC with different backoff ranges in wireless sensor networks," in IEEE ICCS, 2006.

46. A. C. Pang and H. W. Tseng, "Dynamic backoff for wireless personal networks," in IEEE GLOBECOM, 2004.

47. S. C. Ergen, "IEEE 802.15.4 summary," Advanced Technology Lab of National Semiconductor, Tech. Rep., 2004.

48. A. Giridhar and P. R. Kumar, "Toward a theory of in-network computation in wireless sensor networks," IEEE Communication Magazine, pp. 97-107, April 2006.

49. P. Park, P. D. Marco, P. Soldati, C. Fischione, and K. H. Johansson, "A generalized markov chain model for effective analysis of slotted IEEE 802.15.4," in IEEE MASS, 2009.

50. D. P. Bertsekas and J. N. Tsitsiklis, Parallel and Distributed Computation: Numerical Methods. Athena Scientific, 1997.

51. F. Cali, M. Conti, and E. Gregori, "Dynamic tuning of the IEEE 802.11 protocol to achieve a theoretical throughput limit," IEEE/ACM Transactions on Networking, pp. 785 - 799, Dec. 2006.

52. S. C. Ergen, P. D. Marco, and C. Fischione, "MAC protocol engine for sensor networks," in IEEE Globecom, 2009.

53. R. Jain, D. Chiu, and W. Hawe, "A quantitative measure of fairness and discrimination for resource allocation in shared computer systems," Digital Equipment Corporation, Tech. Rep., 1984. 